\title{
Ultrasound-guided epidural anesthesia for a parturient with severe malformations of the skeletal system undergoing cesarean delivery:
}

\section{a case report}

\author{
This article was published in the following Dove Press journal: \\ Local and Regional Anesthesia \\ 6 May 2015 \\ Number of times this article has been viewed
}

\section{LinLi Luo* \\ Juan $\mathrm{Ni}^{*}$ \\ Lan $\mathrm{Wu}$ \\ Dong Luo}

Department of Anesthesiology, West China Second Hospital, Sichuan University, Chengdu, Sichuan,

People's Republic of China

*These authors contributed equally to this work and should be considered co-first authors
Correspondence: Dong Luo

Department of Anaesthesiology,

West China Second Hospital, Sichuan

University, Number 20, Section 3 Renmin

Nan Road, Chengdu, Sichuan 61004I,

People's Republic of China

$\mathrm{Tel}+862885503753$ (office)

Fax +862885503752

Email luodong8706@163.com

\begin{abstract}
Anesthetic management of patients with preexisting diseases is challenging and individualized approaches need to be determined based on patients' complications. We report here a case of ultrasound-guided epidural anesthesia in combination with low-dose ketamine during cesarean delivery on a parturient with severe malformations of the skeletal system and airway problems. The ultrasound-guided epidural anesthesia was performed in the L1-L2 space, followed by an intravenous administration of ketamine $(0.5 \mathrm{mg} / \mathrm{kg})$ for sedation and analgesia. Satisfactory anesthesia was provided to the patient and spontaneous ventilation was maintained during the surgery. The mother and the baby were discharged 5 days after surgery, no complications were reported for either of them. Our work demonstrated that an ultrasound-guided epidural anesthesia combined with low-dose ketamine can be used to successfully maintain spontaneous ventilation and provide effective analgesia during surgery and reduce the risk of postoperative anesthesia-related pulmonary infection.
\end{abstract}

Keywords: anesthesia, regional, cesarean delivery, ketamine, ultrasound-guided

\section{Introduction}

Anesthetic management of patients with severe malformations of the skeletal system can be very challenging due to altered anatomy and physiology. ${ }^{1}$ Here, we report a case of ultrasound-guided epidural block in combination with low-dose ketamine during cesarean section on a patient with severe malformations of the skeletal system and airway problems.

\section{Case report}

The patient signed the informed consent form for this case report. The patient was a 26-year-old woman (height, $142 \mathrm{~cm}$; weight, $42 \mathrm{~kg}$ ) with a gestation of 39 weeks and 3 days. She was selected for the cesarean delivery because of her high-risk pregnancy and spontaneous rupture of membranes.

Physical examination revealed that the patient had anatomic abnormalities including severe cervical spine stiffness, a class 3 Mallampati airway with short thyromental distance and limited mouth opening, severe thoracic lordosis, accentuated lumbar lordosis, deformity of pelvis, right leg length discrepancy, and muscle atrophy in both lower limbs (Figure 1). She also had severely impaired lung functions due to thoracocyllosis and acute bronchitis accompanied with cough and phlegm. At resting state, the respiratory frequency was 30 times/minute. The peripheral oxygen saturation 

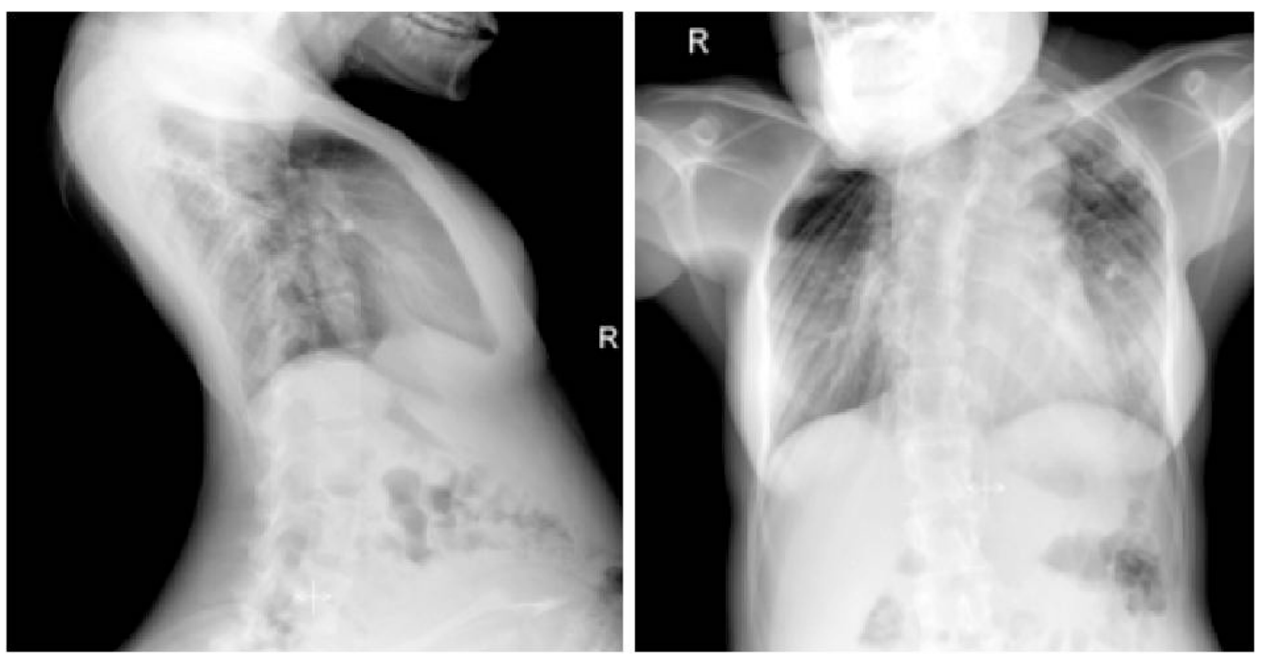

Figure I Lateral (left) and anteroposterior (right) radiographs of patient's thoracic spine and lumbar spine. Note: X-ray showed pectus carinatum, severe thoracic lordosis, accentuated lumbar lordosis, and acute pulmonary infection.

$\left(\mathrm{SpO}_{2}\right)$ by pulse oximetry was $90 \%$ in air and $94 \%$ in $2 \mathrm{~L} /$ minute oxygen through the nasal catheter. The blood pressure, heart rate, and coagulation function were normal, and the fetal safety was confirmed.

Because of the patient's complicated situation, an ultrasound-guided epidural anesthesia in combination with low-dose ketamine was applied. Local anesthesia around incision and low-dose ketamine were the preferred alternate plans. A fiberoptic bronchoscope and an intubation kit for a difficult airway were made available. The patient was maintained on spontaneous breathing with $100 \%$ oxygen via face mask while the epidural anesthesia was performed. Briefly, the patient was placed in the left lateral position, her lumbar spine was scanned using a PhilipsHD11XE portable ultrasound unit (Philips, Bothell, WA, USA) with a $2-5 \mathrm{MHz}$ curved transducer before epidural puncture. With the ultrasound beam oriented in a longitudinal parasagittal plane, it was shown that the L1-L2, L2-L3, and L3-L4 interlaminar spaces were extremely narrow. The L1-L2 intervertebral level was identified and marked on the patient's skin. The distance from the skin to the epidural space was estimated to be approximately $3 \mathrm{~cm}$. A 17-gauge Tuohy needle was inserted into the L1-L2 space, and a catheter was inserted $3 \mathrm{~cm}$ beyond the tip of the needle. A test dose of $3 \mathrm{~mL} \mathrm{1.5 \%} \mathrm{lidocaine} \mathrm{was} \mathrm{administered,}$ followed by two doses of $3 \mathrm{~mL} 1.5 \%$ lidocaine with 5 minute intervals. Sensory block was shown to be thorough within 15 minutes after the last injection of lidocaine, and the upper level of blockage was at the $\mathrm{T} 7$.

Ketamine $(0.5 \mathrm{mg} / \mathrm{kg})$ was then given to the patient intravenously before surgery. Spontaneous ventilation was maintained and $\mathrm{SpO}_{2}$ was kept at 94\%-95\% throughout the surgery. The blood pressure and heart rate were stable during the surgery. The delivery of a 2,980 $\mathrm{g}$ infant with an Apgar score of $9 / 10$ was proceeded uneventfully. The duration of surgery was 35 minutes. The patient's total estimated blood loss was $350 \mathrm{~mL}$ and she received $500 \mathrm{~mL}$ of Ringer solution. The patient recovered smoothly from anesthesia after surgery; there were no signs of memory loss or hallucinations. The epidural catheter was secured in place for patient-controlled epidural analgesia with an infusion of $0.125 \%$ bupivacaine-sufentanil $(0.25 \mu \mathrm{g} / \mathrm{mL})$ at a basal infusion rate of $2 \mathrm{~mL} /$ hour and a $2-\mathrm{mL}$ bolus available for every 30 minutes. One hour after the surgery, the sensory and motor functions of the patient recovered from the neuraxial blockage, and no neurological complications were observed. The patient and the baby were discharged 5 days later; no complications were reported for either the patient or the baby.

\section{Discussion}

The patient in this case report had malformations of the skeletal system in many locations and severely impaired lung functions, making it very difficult in choosing anesthetic approaches.

Spinal malformation is one of the most common diseases causing difficulties in anesthesia, such as difficult intubation. ${ }^{2}$ Airway complications, such as recurrent respiratory infections, upper airway obstruction, and restrictive lung expansion, are also anticipated to be secondary to anatomic anomalies. In a pregnant woman, a greater than normal decrease in functional residual capacity and an increase in closing volume due to restrictive lung disease may promote atelectasis and ventilation/perfusion mismatch. ${ }^{1}$ Moreover, the high 
intra-abdominal pressure in pregnant women increases the risk for aspiration. The supine hypotensive syndrome in the patients with spine malformation can be more severe than that in the normal parturient due to the limited volume replacement. Therefore, anesthetic management of the patients with spine malformation is very challenging.

Although general anesthesia has been recommended for elective cesarean sections in the patients with spinal malformations, ${ }^{3,4}$ general anesthesia was not suitable for this patient because of her multiple complications. First, the fetus and puerpera had lower tolerance to hypoxia. Difficult airway, impaired lung function, and acute bronchitis may lead to severe hypoxemia during general anesthesia. Second, the side effects of general anesthetics on the fetus and the severe stress reaction caused by performing the endotracheal intubation when awake intubation should be considered. Third, the risk of aspiration and pulmonary complications under general anesthesia during pregnancy could be very high. Because the patient in this case report had impaired lung functions, an increased risk of postoperative pulmonary complications and difficulty getting off the ventilator after surgery were expected. Therefore, we did not choose general anesthesia for this patient.

On the other hand, regional anesthesia techniques have been found to be beneficial, especially for patients with difficult airway who are at a high risk for general anesthesia. Under regional anesthesia, the respiratory mechanism remains intact and diaphragm is unaffected; the patient is able to adjust minute ventilation without yielding any significant changes in the ventilatory parameters or $\mathrm{CO}_{2}$ levels. ${ }^{5}$ However, a history of spinal malformations might increase the difficulty of puncture. The anatomic abnormity of the subarachnoid space can result in an unpredictable anesthetic spread and a high risk of spinal cord injury. ${ }^{6}$ Furthermore, severe inhibition of respiration and circulation due to the high anesthesia level resulting from a combined spinal-epidural or a single-shot spinal anesthesia will also be dangerous to the patient. Additionally, hypotension may decrease uteroplacental perfusion and cause fetal acidosis. The routine volume replacement for preventing hypotension in puerpera before spinal anesthesia ${ }^{7}$ will also be risky in the patient in this case report. She may not be able to tolerate the rapid volume change because she had cardiopulmonary compression, lower left atelectasis, and lung inflammation. Therefore, a combined spinal-epidural or a single-shot spinal anesthetic technique was not chosen for the patient. However, a continuous spinal anesthesia technique might be a good option. A very small dose of local anesthetic can be injected intrathecally and slowly titrated to achieve the desired level of block. Excellent motor and sensory block can be achieved, and the level of the block can be easily controlled. Since we did not have the specialized tool for a continuous spinal anesthesia, this technique was not chosen for this patient. Epidural anesthesia was chosen as the optimal option for this patient. Epidural anesthesia had fewer side effects on both the patient and the baby. It has a predictable dose spread in the epidural space and has fewer side effects on respiratory and circulatory systems than general and spinal anesthesia do. However, the potential increased risk of difficult puncture, difficult catheter placement, failed epidural block, and other complications must all be taken into consideration before any epidural puncture is applied. ${ }^{1}$ An MRI examination should be considered before the operation to identify the anatomic change of canalis vertebralis and medulla spinalis. Ultrasound scan can provide a lot of anatomic information pertinent to central neuraxial blockade ${ }^{8}$ and allow surgeons to formulate an effective anesthetic management plan, which has been considered to improve the success rate and lower the complications in the central neuraxial blockade when technical difficulty is anticipated..$^{9,10}$ Therefore, ultrasound-guided epidural anesthesia was selected for this patient. We used the ultrasound as guidance for the puncture, and this was our first case using ultrasound to aid anesthesia. Unfortunately, the ultrasound images were not taken during the procedure.

Because of the patient's complex situation, it was difficult to determine the adequate volume and dose needed for the surgical procedure. Lidocaine $(1.5 \%, 9 \mathrm{~mL})$ was titrated to meet the basic demand of anesthesia for this patient. Although a T7 block may not be adequate for cesarean delivery in most patients, considering the multiple and complex complications the patient had before the surgery, we chose a low block level of $\mathrm{T} 7$ to avoid the risk of further inhibition of respiration and circulation, and we used ketamine for supplemental analgesia.

Sedatives and analgesic drugs are often used in epidural block for a satisfactory anesthesia. Impaired lung functions may increase the patient's sensibility to sedatives and the side effects of analgesic drugs associated with the respiratory system. Spinal and epidural anesthesia can cause changes in the circulatory system due to insufficient capacity of circulation, sympathetic block, and a decrease in systemic vascular resistance. These side effects, however, can be partially reversed by ketamine, which has unique central sympathomimetic, vagolytic, and analgesic properties. When used at anesthetic doses, ketamine will usually stimulate rather than depress the respiratory and circulatory systems; it will help to preserve the upper airway muscle, laryngopharyngeal 
reflexes, bronchodilator effect, and protective reflexes; it will also help to maintain the spontaneous ventilation and keep the airway unobstructed during the entire course of anesthesia. ${ }^{11,12}$ As an anesthetic, ketamine was rarely used alone except for critical care conditions. Ketamine at low dose $(0.2-0.5 \mathrm{mg} / \mathrm{kg})$ has been increasingly used to complement other anesthetics in sedation and analgesia. ${ }^{12,13}$ Therefore, an intravenous injection of ketamine $(0.5 \mathrm{mg} / \mathrm{kg})$ was applied following epidural anesthesia for this patient. We have recognized the side effects of ketamine, such as hallucinations, that can occur even at small doses, so the patient was closely monitored during the intraoperative and postoperative period.

In summary, we discussed the advantages and disadvantages of several anesthetic techniques and successfully developed an ultrasound-guided epidural anesthesia method combined with low-dose ketamine based on the patient's complications. In future, individualized approaches should be considered for anesthetic management of high-risk pregnant patients with complex and multiple medical and surgical morbidities. It is essential to have a multidisciplinary team approach for high-risk pregnant patients. This team should consist of a general surgeon, critical care specialists, radiologists, anesthesiologists, and obstetricians. This team approach and the peripartum management of such patients should be planned ahead of the scheduled surgery.

\section{Disclosure}

The authors report no conflicts of interest in this work.

\section{References}

1. DeRenzo JS, Vallejo MC, Ramanathan S. Failed regional anesthesia with reduced spinal bupivacaine dosage in a parturient with achondroplasia presenting for urgent cesarean section. Int J Obstet Anesth. 2005;14: $175-178$.
2. Leung KH, Chiu KY, Wong YW, Lawmin JC. Case report: spinal anesthesia by mini-laminotomy for a patient with ankylosing spondylitis who was difficult to anesthetize. Clin Orthop Relat Res. 2010;468: 3415-3418.

3. Cohen V, Powell E, Lake C. Failure of neuraxial anaesthesia in a patient with velocardiofacial syndrome. Int J Obstet Anesth. 2011;20: 256-259.

4. Olufolabi AJ, Wee MY. Caesarean section in a patient with torsion dystonia. Br J Anaesth. 2006;96:611-613.

5. van Zundert AA, Stultiens G, Jakimowicz JJ, van den Borne BE, van der Ham WG, Wildsmith JA. Segmental spinal anaesthesia for cholecystectomy in a patient with severe lung disease. Br J Anaesth. 2006;96:464-466.

6. van Bogaert LJ. Lumbar lordosis and the spread of subarachnoid hyperbaric $0.5 \%$ bupivacaine at cesarean section. Int J Gynaecol Obstet. 2000;71:65-66.

7. Dyer RA, Farina Z, Joubert IA, et al. Crystalloid preload versus rapid crystalloid administration after induction of spinal anaesthesia (coload) for elective caesarean section. Anaesth Intensive Care. 2004;32: 351-357.

8. Chin KJ, Perlas A, Singh M, et al. An ultrasound-assisted approach facilitates spinal anesthesia for total joint arthroplasty. Can J Anaesth. 2009;56:643-650.

9. Sahota JS, Carvalho JC, Balki M, Fanning N, Arzola C. Ultrasound estimates for midline epidural punctures in the obese parturient: paramedian sagittal oblique is comparable to transverse median plane. Anesth Analg. 2013;116:829-835.

10. Chin KJ, Chan V. Ultrasonography as a preoperative assessment tool: predicting the feasibility of central neuraxial blockade. Anesth Analg. 2010;110:252-253.

11. Eikermann M, Grosse-Sundrup M, Zaremba S, et al. Ketamine activates breathing and abolishes the coupling between loss of consciousness and upper airway dilator muscle dysfunction. Anesthesiology. 2012;116:35-46.

12. Canet J, Castillo J. Ketamine: a familiar drug we trust. Anesthesiology. 2012;116:6-8.

13. Mukhopadhyay S, Niyogi M, Dutta M, et al. Bilateral superficial cervical plexus block with or without low-dose intravenous ketamine analgesia: effective, simple, safe, and cheap alternative to conventional general anesthesia for selected neck surgeries. Local Reg Anesth. 2012;5:1-7.
Local and Regional Anesthesia

\section{Publish your work in this journal}

Local and Regional Anesthesia is an international, peer-reviewed, open access journal publishing on the development, pharmacology, delivery and targeting and clinical use of local and regional anesthetics and analgesics. The journal welcomes submitted papers covering original research, basic science, clinical studies, reviews \& evaluations,

\section{Dovepress}

guidelines, expert opinion and commentary, case reports and extended reports. The manuscript management system is completely online and includes a very quick and fair peer-review system, which is all easy to use. Visit http://www.dovepress.com/testimonials.php to read real quotes from published authors. 